\title{
Interference with spatial working memory: An eye movement is more than a shift of attention
}

\author{
BONNIE M. LAWRENCE \\ Washington University School of Medicine, St. Louis, Missouri \\ and \\ JOEL MYERSON and RICHARD A. ABRAMS \\ Washington University, St. Louis, Missouri
}

\begin{abstract}
In the present experiments, we examined whether shifts of attention selectively interfere with the maintenance of both verbal and spatial information in working memory and whether the interference produced by eye movements is due to the attention shifts that accompany them. In Experiment 1, subjects performed either a spatial or a verbal working memory task, along with a secondary task requiring fixation or a secondary task requiring shifts of attention. The results indicated that attention shifts interfered with spatial, but not with verbal, working memory, suggesting that the interference is specific to processes within the visuospatial sketchpad. In Experiment 2, subjects performed a primary spatial working memory task, along with a secondary task requiring fixation, an eye movement, or an attention shift executed in the absence of an eye movement. The results indicated that both eye movements and attention shifts interfered with spatial working memory. Eye movements interfered to a much greater extent than shifts of attention, however, suggesting that eye movements may contribute a unique source of interference, over and above the interference produced by the attention shifts that accompany them.
\end{abstract}

Working memory is a cognitive system for the shortterm maintenance and manipulation of information. This system is commonly conceptualized as consisting of three components: a phonological loop for maintaining verbal information, a visuospatial sketchpad for maintaining visuospatial information, and a central executive for coordinating information within the working memory system (Baddeley, 1986). An important strategy for investigating each component's function has been to determine which cognitive and behavioral processes interfere with which components and which do not. However, research based on this strategy has tended to focus on the maintenance of verbal information, and as a result, much less is known about what interferes with the maintenance of visuospatial information.

The present experiments concern the maintenance of spatial information in the visuospatial sketchpad. Previous research has demonstrated that both eye movements and arm movements interfere with spatial working memory (Hale, Myerson, Rhee, Weiss, \& Abrams, 1996; Lawrence, Myerson, Oonk, \& Abrams, 2001). Because both eye and

This research was supported by Grant BCS-0079594 from the National Science Foundation to R.A.A. and by a Grants-in-Aid of Research Award from Sigma Xi, awarded to B.M.L. We thank Larry H. Snyder for comments on an early version of the article. Correspondence should be addressed to B. M. Lawrence, Department of Anatomy and Neurobiology, Washington University School of Medicine, Box 8108, 660 South Euclid Ave., St. Louis, MO 63110 (e-mail: bonnie@eye-hand.wustl.edu). arm movements are accompanied by shifts of attention (see, e.g., Boulinguez \& Nougier, 1999; Shepherd, Findlay, \& Hockey, 1986), it is possible that the interference produced by such movements is due to these attention shifts.

Consistent with this hypothesis, Smyth (1996, Experiment 1) reported that discriminating the spatial location of the source of an auditory tone and, presumably, shifting attention in order to do so interfered with the maintenance of spatial information. However, executive processes required for the discriminations, and not the shifts of attention, may have been the source of interference in Smyth's experiment (Klauer \& Stegmaier, 1997). More recently, Awh, Jonides, and Reuter-Lorenz (1998, Experiment 3) reported that shifting attention away from a tobe-remembered spatial location resulted in a decrement in the accuracy with which that location could be recalled. Awh et al. required subjects to remember only one location at a time, however, and it is unclear whether the mechanisms involved in remembering a single location are the same as those involved in maintaining multiple locations in working memory. Moreover, Awh et al. did not determine whether attention shifts interfere with working memory processes in general or whether attention shifts interfere with processes underlying the maintenance of information in spatial working memory in particular.

Experiment 1 addressed these issues by examining whether or not shifts of attention would interfere with the maintenance of multiple items in spatial and verbal work- 
ing memory. If shifts of attention were to interfere with spatial, but not with verbal, working memory, this would suggest that the source of the interference produced by attention shifts may be localized to processes specific to the visuospatial sketchpad (Baddeley, 1986). As will be seen, the results of Experiment 1 indicated that attention shifts selectively interfered with spatial working memory. As was previously noted, goal-directed movements (e.g., eye and arm movements) also selectively interfere with spatial working memory, and this may be because such movements are accompanied by shifts of attention (e.g., Hale et al., 1996; Lawrence et al., 2001; Smyth, 1996; Smyth \& Scholey, 1994). Experiment 2 addressed this issue by comparing the amount of interference produced by shifts of attention with the amount of interference produced by eye movements.

\section{EXPERIMENT 1}

The purpose of Experiment 1 was to determine whether or not shifts of attention would interfere with the maintenance of multiple spatial locations in working memory and whether attention shifts would also interfere with verbal working memory. The spatial working memory task required subjects to remember a series of locations, whereas the verbal working memory task required subjects to remember a series of letters. These two working memory tasks were crossed with two secondary tasks: a no-shift secondary task requiring fixation and the discrimination of stimuli appearing at fixation and an attention shift task requiring fixation and the discrimination of stimuli appearing in the periphery.

\section{Method}

Subjects. Twenty-four undergraduate students from Washington University completed the experiment in a 1.5 -h session for course credit.

Apparatus and Procedure. The experiment was conducted in a dimly lit room. Stable head position and constant viewing distance (i.e., $55 \mathrm{~cm}$ from the computer monitor) were maintained using a chinrest. The primary working memory tasks were accompanied either by a secondary task requiring fixation and a discrimination (no-shift condition) or by a secondary task requiring shifts of attention and a discrimination (attention shift condition).

The stimuli for the spatial working memory task consisted of a series of blue circles $\left(1.25^{\circ}\right.$ in diameter), each presented for $2,000 \mathrm{msec}$ in one of 20 cells of a white cross-shaped grid $\left(4.7^{\circ} \times\right.$ $\left.7.8^{\circ}\right)$ located in the center of the screen. The locations of the circles were selected randomly without replacement for each series in such a way that no location could be cued more than once in a series. The centrally located cell was filled white and could not contain a memory item (i.e., a circle). This was because a discrimination stimulus could appear in that same location during the secondary task. The subjects were instructed to remember the locations of the circles as accurately as possible. At the end of each series, an empty grid appeared, which served as a signal to recall the memory items. Recalled items were reported by moving the mouse cursor to the remembered locations, clicking the left mouse button once in each location. The subjects signaled the completion of the recall period by clicking once in the space outside of the grid. A tone was sounded if the subjects did not correctly recall all of the memory items.

The stimuli for the verbal working memory task consisted of a series of yellow letters $\left(0.6^{\circ} \times 0.6^{\circ}\right)$, each presented at fixation for
2,000 msec. For each verbal working memory series, letters were selected randomly and without replacement from the following list: B, D, F, G, H, J, K, L, M, N, Q, R, S, T, W, and X. Vowels were not included in the list of letters in order to minimize the likelihood that the subjects would remember the list of letters as a word. The subjects were instructed to remember the identity and order of the letters as accurately as possible.

At the end of each series, a $4 \times 4$ light gray grid $\left(6.25^{\circ} \times 6.25^{\circ}\right)$ appeared containing the list of possible memory items (presented in white) in alphabetical order from left to right in the cells of the grid. This grid signaled the subjects to recall the memory items by moving a mouse cursor to each of the previously displayed letters and clicking the left mouse button once on each letter. When the subjects clicked on a letter, the letter turned from white to green, and the grid space surrounding the letter in a grid cell turned from dark gray to light gray. Thus, the subjects had a visual cue as to the letter or letters they had selected. The subjects signaled the completion of the recall period by clicking once in the space outside of the grid. A tone was sounded if the subjects did not correctly recall the memory items.

The number of memory items (i.e., the series length) was determined by a modified version of the WAIS-III memory span procedure (Psychological Corporation, 1997). As in the WAIS-III procedure, the subjects were given two trials at each series length until they missed two trials at any one series length. Thus, the number of trials depended on the subjects' performance. Unlike in the WAIS-III procedure, when the subjects missed two trials at any one series length, they were given one last trial at a series length of one item less. If the subject was correct on this trial, his or her span was determined to be the length of the series in this trial. If, however, the subject was incorrect on this trial, the span was determined to be this series length minus one half an item. Each condition began with a series of two memory items.

Secondary task stimuli were interleaved between the presentations of each of the working memory items (i.e., circles or letters), as opposed to being presented simultaneously with the primary memory items, so that completion of the secondary task would not interfere with encoding of the primary task items. The stimuli for the secondary task consisted of the consecutive presentation of two stimuli (white Xs or $+\mathrm{s}$ ). The stimuli were randomly chosen and could be either the same (e.g., two Xs) or different (e.g., an X and $\mathrm{a}+$ ). The first stimulus was presented for $500 \mathrm{msec}$. Two hundred milliseconds after the offset of the first stimulus, the second stimulus was presented for $1,800 \mathrm{msec}$, at which time the subjects were to click the left mouse button if the identity of the stimuli was the same or the right mouse button if the identity of the stimuli was different. An error message was given if the subjects pressed the incorrect mouse button. In the no-shift condition, the discrimination stimuli appeared in the center of the screen, where the subjects were instructed to maintain fixation. In the attention shift condition, the discrimination stimuli appeared $9.6^{\circ}$ to either the left or the right of fixation, and the order of appearance (left-right or right-left) was randomly selected. In both the no-shift and the attention shift conditions, the subjects were instructed to maintain fixation on the center of the screen throughout the trial.

Example trials for the spatial and the verbal working memory tasks with secondary task conditions are presented in Figures 1 and 2. Note that for the sake of brevity, the figures depict trials in which only one memory item was presented, and for simplicity, the figures depict black stimuli presented on a white background, as opposed to the actual experiment, in which both white and colored stimuli were presented on a dark gray background. For all the conditions, a trial began with a fixation point $(500 \mathrm{msec})$, followed by the presentation of a circle $(2,000 \mathrm{msec})$ in one of the locations of a grid for the spatial working memory task (Figure 1) or by the presentation of a letter $(2,000 \mathrm{msec})$ for the verbal working memory task (Figure 2).

The secondary task stimuli (fixation points alternating with discrimination stimuli consisting of Xs and $+\mathrm{s}$ ) were then presented. 


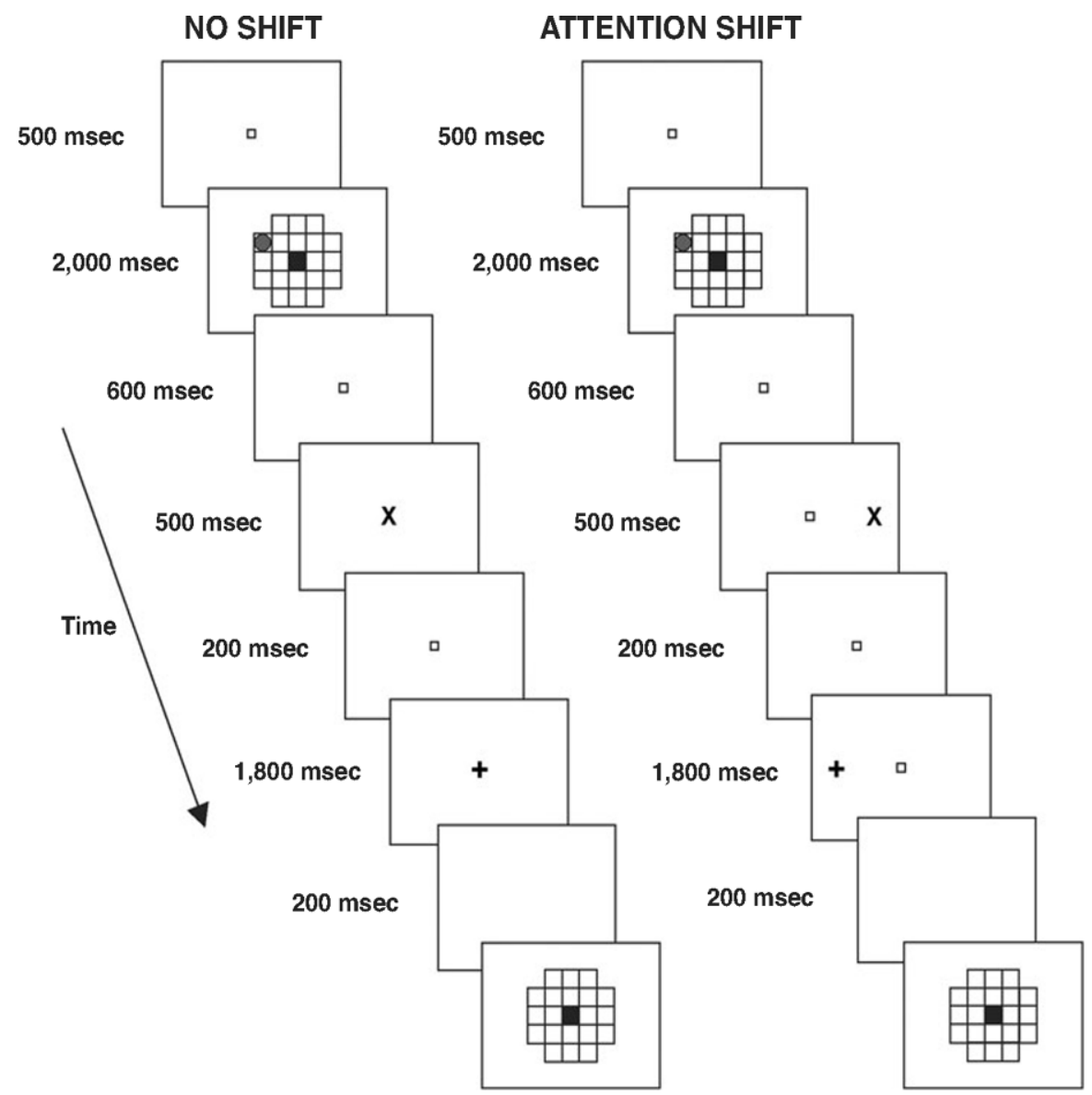

Figure 1. The timing of stimulus presentation for the spatial working memory conditions in Experiment 1 .

More specifically, the secondary task stimuli began with a fixation point $(600 \mathrm{msec})$, followed by a discrimination stimulus $(500 \mathrm{msec})$, by another fixation point $(200 \mathrm{msec})$, and then by another discrimination stimulus $(1,800 \mathrm{msec})$. This was followed by a blank screen (200 msec), after which an empty grid (Figure 1) or a letter grid (Figure 2) was presented to signal the recall of the memory items. On multi-item trials, each memory item was followed by secondary stimuli, with the signal to recall appearing only after the final discrimination stimulus. In the no-shift condition, the discrimination stimuli appeared at fixation, whereas in the attention shift condition, the discrimination stimuli appeared in the periphery.

Eye movement monitoring. Stimulus presentation was computer controlled, as was the acquisition of eye movement data. Eye position was monitored using an ISCAN (Cambridge, MA) videobased eye movement monitor. The eye movement monitor was calibrated at the beginning of each experimental condition by presenting stimuli at five evenly spaced points across the display and requiring the subjects to fixate each of these points.

Eye position was recorded at a rate of $60 \mathrm{~Hz}$ for $2,500 \mathrm{msec}$, beginning with the offset of each memory item. In order to identify saccades, the eye position signal was filtered and differentiated so that a smooth velocity record could be obtained. The beginning of an eye movement was defined as the first moment in time at which the velocity exceeded $10 \mathrm{deg} / \mathrm{sec}$ for at least $32 \mathrm{msec}$, with the constraint that velocity subsequently exceeded $30 \mathrm{deg} / \mathrm{sec}$.

In all the conditions, an error message was presented if the subjects were not fixating on the center of the screen after the presentation of the memory items and prior to the presentation of the sec- ondary task stimulus (see Figure 1). An error message was also presented if blinks, sufficient to disrupt the monitoring of eye position, occurred or if an eye movement was made between memory items when the subjects were supposed to be fixating on the center of the screen. If one of the preceding errors occurred, the memory trial was aborted and immediately rerun at the same series length.

Design. The subjects participated in all four blocked conditions (spatial no shift, spatial attention shift, verbal no shift, and verbal attention shift). The presentation of the working memory conditions (i.e., spatial or verbal) was counterbalanced across subjects. Within a working memory condition, the secondary task conditions (i.e., no shift or attention shift) were counterbalanced across subjects.

Prior to the beginning of each working memory condition (i.e., spatial or verbal), the subjects were required to correctly complete six practice trials of each working memory task. The practice trials consisted of two trials each at series lengths of one, two, and three. These working memory trials were not accompanied by a secondary task. These trials were included to ensure that the subjects could maintain three spatial and three verbal items in working memory. In addition, the subjects completed six practice trials with the appropriate secondary task (no shift or attention shift) prior to each block.

\section{Results and Discussion}

A 2 (domain: spatial or verbal) $\times 2$ (condition: no shift or attention shift) repeated measures analysis of variance (ANOVA) conducted on eye movement errors 


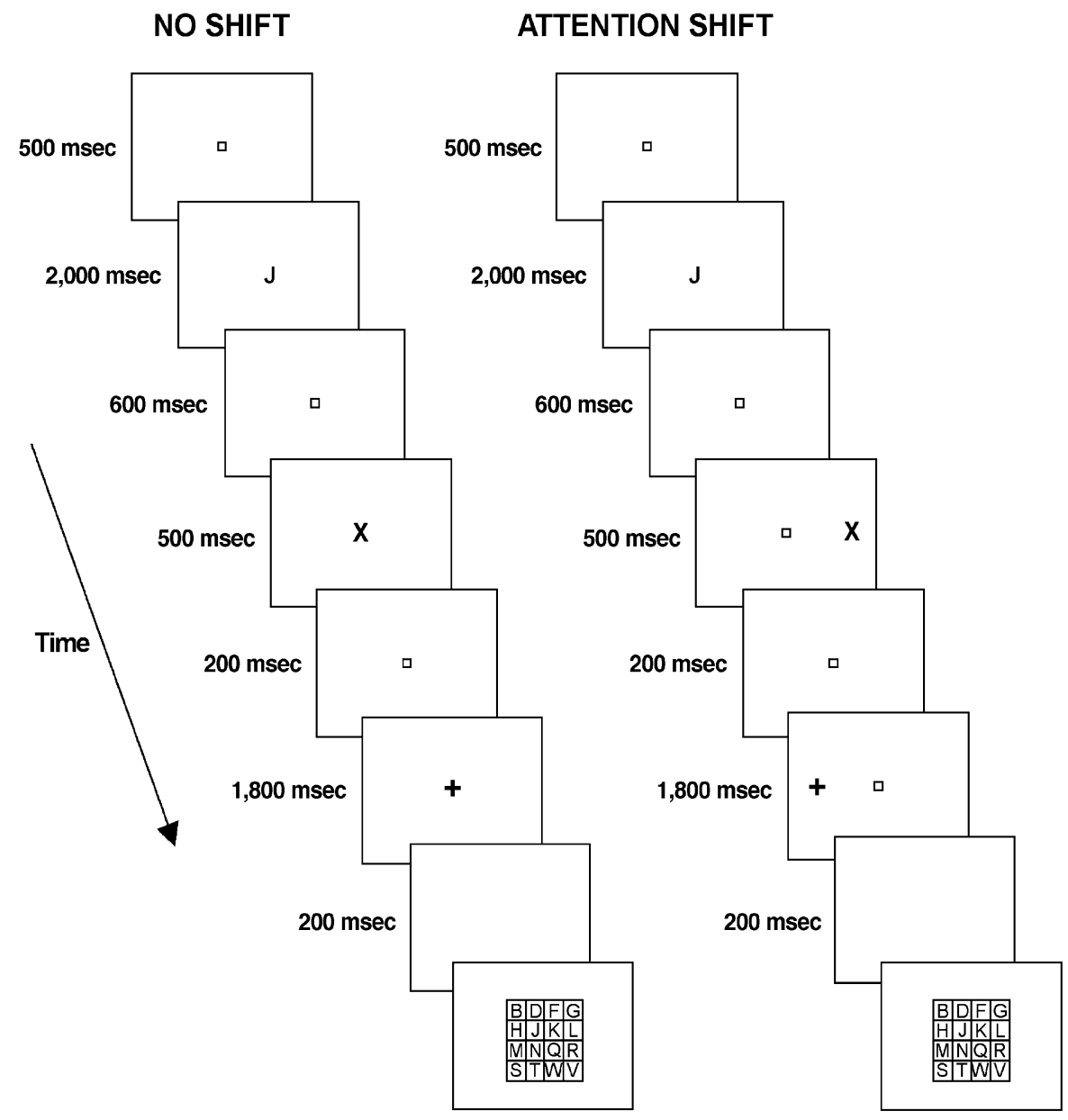

Figure 2. The timing of stimulus presentation for the verbal working memory conditions in Experiment 1.

indicated that there were no significant effects of either condition or domain and no significant interaction [all $F \mathrm{~s}(1,23)<1.2$; mean errors for the spatial no-shift, spatial attention shift, verbal no-shift, and verbal attention shift conditions were $5.29,4.75,5.00$, and 6.58 , respectively; these values include blinks, as well as failures to maintain fixation].

Mean memory spans (i.e., the maximum number of items that a subject could correctly recall) for the spatial no-shift $(M=5.92)$, the spatial attention shift $(M=$ 4.31), the verbal no-shift $(M=6.96)$, and the verbal attention shift $(M=6.69)$ conditions are presented in Figure 3. A 2 (domain: spatial or verbal) $\times 2$ (condition: no shift or attention shift) repeated measures ANOVA was conducted on the memory spans. The results revealed main effects of both domain $[F(1,23)=30.35, p<.001]$ and condition $[F(1,23)=9.42, p<.01]$, as well as a significant domain $\times$ condition interaction $[F(1,23)=10.67$, $p<.05]$, reflecting the fact that the difference between the shift and the no-shift conditions was greater in the spatial domain. 1

Planned comparisons revealed that there was a significant difference between the no-shift and the attention shift conditions in the spatial domain $[t(23)=3.91, p<$ $.005]$ but that there was no significant difference between the no-shift and the attention shift conditions in the verbal domain $[t(23)<1]$. Importantly, the same discrimination between two nameable shapes (an X and a + ) was used for both the no-shift and the attention shift secondary task conditions. The domain $\times$ condition interaction, therefore, cannot have been due to the nature of the discrimination. Although some caution in interpretation may be indicated because of the difference between verbal and spatial spans in the baseline no-shift conditions, we believe that the observed interaction reflects selective interference with spatial working memory being produced by spatial attention shifts, suggesting that spatial attention is necessary for the maintenance of information in spatial, but not verbal, working memory.

\section{EXPERIMENT 2}

The results of Experiment 1 suggest that shifts of attention selectively interfere with spatial working memory. This finding is consistent with the suggestion that the interference produced by goal-directed movement is 


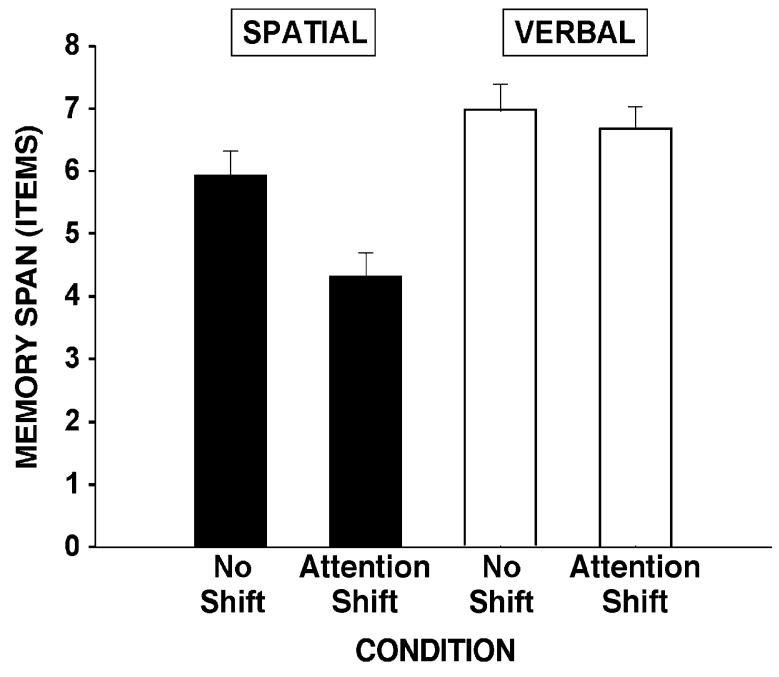

Figure 3. The results of Experiment 1 (mean memory spans and standard errors) for the spatial no-shift, spatial attention shift, verbal no-shift, and verbal attention shift conditions, indicating that shifts of attention interfered with spatial, but not verbal, working memory.

not the result of the execution of the movements themselves but, rather, is due to the shifts of attention that accompany them (e.g., Hale et al., 1996; Lawrence et al., 2001; Smyth, 1996; Smyth \& Scholey, 1994). The purpose of the present experiment was to determine whether the interference produced by shifts of attention would be equivalent in magnitude to that produced by eye movements. If shifts of attention alone underlie the maintenance of information in spatial working memory, and if eye movements are accompanied by shifts of attention (e.g., Shepherd et al., 1986), shifts of attention and eye movements should interfere to an equivalent extent. If the interference produced by eye movements cannot be explained simply by shifts of attention, however, eye movements should interfere to a greater extent than shifts of attention.

In the present experiment, the spatial working memory task required the subjects to remember a series of spatial locations. Following the presentation of each of the spatial locations, the subjects executed one of the following secondary tasks: a no-shift secondary task that required maintaining fixation and identifying a visual stimulus presented at fixation, an attention shift secondary task that required maintaining fixation and identifying a visual stimulus presented at one of two peripheral locations, or an eye movement secondary task that required making an eye movement to a visual stimulus presented at one of two peripheral locations and then discriminating the identity of this stimulus. Note that the procedures for the eye movement and the attention shift conditions differed only with respect to the fact that the former required an eye movement, whereas the latter required maintaining fixation.

\section{Method}

Subjects. Thirty undergraduate students from Washington University completed the experiment in a 1-h session for a payment of $\$ 10$.
Apparatus and Procedure. The experiment was conducted in a dimly lit room. A chinrest was used to maintain stable head position and constant viewing distance (i.e., $55 \mathrm{~cm}$ from the computer monitor). The presentations of the stimuli for the primary spatial working memory task were interleaved with a secondary task requiring fixation and a discrimination (no shift), a secondary task requiring a shift of attention and a discrimination (attention shift), or a secondary task requiring an eye movement and a discrimination (eye movement). The background color was dark gray throughout all the conditions.

The secondary task discrimination stimulus consisted of either a white $\mathrm{X}\left(0.63^{\circ}\right)$ or a white $+\left(0.63^{\circ}\right)$, which appeared for $2,500 \mathrm{msec}$ in all the conditions. Each discrimination stimulus had a $50 \%$ probability of appearing. The subjects were instructed to click the left mouse button once if an X appeared and twice if a + appeared. A tone was sounded if the subject pressed incorrectly, but the trial proceeded. In the no-shift condition, the discrimination stimulus appeared in the center of the screen, where the subjects were instructed to maintain fixation. In the attention shift and eye movement conditions, the discrimination stimulus appeared $6.45^{\circ}$ to either the left or the right of screen center. In the attention shift condition, the subjects were instructed to maintain fixation on the center of the screen. In the eye movement condition, the subjects were instructed to move their eyes to the discrimination stimulus and back to screen center. Error messages were presented if the subject's behavior did not meet these criteria (see the Method section for Experiment 1).

Eye movement monitoring. The apparatus and procedure for monitoring eye movements were the same as those described in Experiment 1. In all the conditions, as in Experiment 1, an error message was presented if blinks disrupted the monitoring of eye position. In the eye movement condition, an error message was presented if an eye movement occurred too soon (latency less than $100 \mathrm{msec}$ ) or too late (latency greater than $700 \mathrm{msec}$ ), was in the opposite direction from that cued by the peripheral item, or was not executed.

Design. All the subjects participated in each of the three separately blocked conditions (no shift, attention shift, and eye movement). The order of conditions was completely counterbalanced across subjects. The subjects received six practice trials prior to each condition, consisting of two trials at series lengths of one, two, and three.

\section{Results and Discussion}

A one-way repeated measures ANOVA conducted on the eye movement errors from the three conditions indicated that there were no significant differences in the mean number of eye movement errors $[F(2,56)=2.42$, n.s.; mean errors for the no-shift, attention shift, and eye movement conditions were 5.10, 5.03, and 3.31, respectively]. Secondary task accuracy was greater than $98 \%$ in all the conditions. A one-way repeated measures ANOVA conducted on secondary task accuracy from the three conditions indicated that there was not a significant difference in accuracy across conditions $[F(2,56)<1.0$, n.s. $]$. Thus, condition differences in memory spans are not attributable to differences in secondary task performance.

Mean memory spans and standard errors for each task condition are presented in Figure 4. A one-way repeated measures ANOVA conducted on memory spans indicated a significant main effect of condition $[F(2,58)=$ $17.02, p<.001]$. Memory spans were highest in the fixation condition $(M=5.68)$ and lowest in the eye movement condition $(M=3.50)$. Spans in the attend condition $(M=4.78)$ were intermediate between those in the other two conditions. Planned comparisons revealed a 


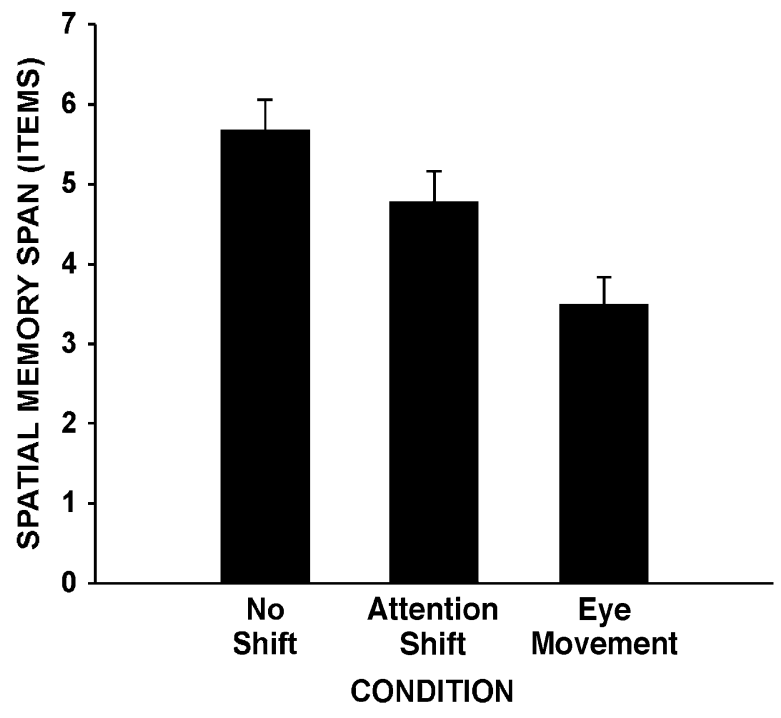

Figure 4. The results of Experiment 2 (mean memory spans and standard errors) for the no-shift, attention shift, and eye movement conditions, indicating that the interference produced by eye movements was significantly greater than the interference produced by shifts of attention alone.

significant difference in memory span between the fixation condition and the attend condition $[t(29)=2.28$, $p<.05]$ and a significant difference in memory span between the attend condition and the eye movement condition $[t(29)=3.79, p<.01]$.

The results of Experiment 2 indicate that although shifts of attention and eye movements interfere with spatial working memory, the amount of eye movement interference was significantly greater than the amount of attention shift interference. These findings suggest that, in addition to the interference produced by the shifts of attention that accompany eye movements, the eye movements themselves may constitute a unique source of interference.

\section{GENERAL DISCUSSION}

The results of Experiment 1 demonstrate that shifts of attention interfere with the maintenance of information in spatial working memory, consistent with the interpretation of previous studies by Smyth (1996) and Awh et al. (1998). The present results demonstrate further that the interference produced by attention shifts, like the interference produced by eye movements (Lawrence et al., 2001), is domain specific, affecting spatial, but not verbal, working memory. This finding suggests that the interference produced by attention shifts, like that produced by eye movements, is localized in the visuospatial sketchpad and is not the consequence of the disruption of general processes involved in the maintenance of both verbal and spatial information.

The results of Experiment 2 demonstrate that although shifts of attention interfere with the maintenance of information in spatial working memory, the execution of eye movements produces significantly more interference than do shifts of attention alone. In fact, the amount of interference in the eye movement condition was more than twice that in the attend condition. This finding suggests that interference associated with the execution of eye movements cannot be explained simply in terms of shifts of attention.

The logic underlying this conclusion implicitly assumes that the shifts of attention that accompany eye movements are the same as the shifts of attention executed in the absence of eye movements. It is possible, however, that the attention shifts that accompany eye movements have different temporal or spatial properties. In Experiment 2, for example, attention may have been shifted to the peripheral location for a longer amount of time on eye movement trials than on attention shift trials, resulting in a longer disruption of rehearsal processes and, hence, greater interference in the eye movement condition. It is also possible that the spatial extent of the attention shift may have been more focused on eye movement trials (Eriksen \& St. James, 1986), although it is less clear how the latter would affect the magnitude of interference with spatial working memory.

For the present, we favor an explanation of the additional interference produced by eye movements as being the result of the eye movements themselves, rather than the result of temporal or spatial differences in attention shifts. Two possible sources of the additional interference produced by eye movements are changes in the retinal coordinates of to-be-remembered locations and the cognitive suppression of spatial processing thought to occur during the execution of eye movements (Irwin \& Brockmole, 2000). Neither of these potential interference sources is present in attention shifts that occur in the absence of eye movements, which may be the reason eye movements produce greater interference.

The results of previous experiments (e.g., Hale et al., 1996; Lawrence et al., 2001) indicate that both eye movements and arm movements interfere with the maintenance of information in spatial working memory. Given that both eye and arm movements are accompanied by shifts of attention (e.g., Boulinguez \& Nougier, 1999; Shepherd et al., 1986), the present finding that attention shifts also interfere with spatial working memory suggest that the interference produced by goal-directed movements are due, in part, to shifts of attention. Importantly, the present results reveal that eye movements produce additional interference over and above that produced by attention shifts, suggesting that the interference produced by goal-directed movement cannot by explained simply by the shifts of attention that accompany them.

\section{REFERENCES}

Awh, E., Jonides, J., \& Reuter-Lorenz, P. A. (1998). Rehearsal in spatial working memory. Journal of Experimental Psychology: Human Perception \& Performance, 24, 780-790.

BADDELEY, A. D. (1986). Working memory. Oxford: Oxford University Press. 
Boulinguez, P., \& Nougier, V. (1999). Control of goal-directed movements: The contribution of orienting of visual attention and motor preparation. Acta Psychologica, 103, 21-45.

ERIKSEN C. W., \& ST. James, J. D. (1986). Visual attention within and around the field of focal attention: A zoom lens model. Perception \& Psychophysics, 40, 225-240.

Hale, S., Myerson, J., Rhee, S. H., Weiss, C. S., \& Abrams, R. A. (1996). Selective interference with the maintenance of location information in working memory. Neuropsychology, 10, 228-240.

IRWIN, D. E., \& BRocKmole, J. R. (2000). Mental rotation is suppressed during saccadic eye movements. Psychonomic Bulletin \& Review, 7 , 654-661.

Jenkins, L., Myerson, J., Hale, S., \& Fry, A. F. (1999). Individual and developmental differences in working memory across the life span. Psychonomic Bulletin \& Review, 6, 28-40.

KLAUER, K. C., \& STEgmaiER, R. (1997). Interference in immediate spatial memory: Shifts of spatial attention or central-executive involvement? Quarterly Journal of Experimental Psychology, 50A, 79-99.

Lawrence, B. M., Myerson, J., Oonk, H. M., \& Abrams, R. A. (2001). The effects of eye and limb movements on working memory. Memory, 9, 433-444.

PsÝCHOLOGICAL CoRPoRATION (1997). Wechsler Adult Intelligence Scale-III: Administration and scoring manual. San Antonio: Harcourt Brace.
Shepherd, M., Findlay, J. M., \& Hockey, R. J. (1986). The relationship between eye movements and spatial attention. Quarterly Journal of Experimental Psychology, 38A, 475-491.

SмYтн, M. M. (1996). Interference with rehearsal in spatial working memory in the absence of eye movements. Quarterly Journal of Experimental Psychology, 49A, 940-949.

SMyth, M. M., \& Scholey, K. A. (1994). Interference in immediate spatial memory. Memory \& Cognition, 22, 1-13.

\section{NOTE}

1. It might be argued that the secondary task discrimination is more spatial than verbal in nature and that the resulting cross-talk underlies the observed lower spatial than verbal spans in the no-shift conditions. However, we do not believe this to be the case, because memory span procedures such as those used in the present study frequently result in smaller spatial than verbal spans even when there is no secondary task requirement (Jenkins, Myerson, Hale, \& Fry, 1999).

(Manuscript received August 30, 2002; revision accepted for publication March 18, 2003.) 